\title{
THE TANGENTIAL GAS INJECTION AS THE MEANS OF HEAT PROTECTION OF A BLUNT BODY STREAMLINED BY DUSTED GAS
}

\author{
E. B. Vasilevskiy and L. V. Yakovleva \\ Central Aerohydrodynamic Institute (TsAGI) \\ Zhukovsky Str. 1, Zhukovsky, Moscow region 140180, Russia
}

The experimental study of a tangential gas injection influence on the heat flux was carried out. Experiments were conducted in TsAGI wind tunnel at free-stream Mach number $\mathrm{M}_{\infty}=6$, Reynolds number $\operatorname{Re}_{\infty, R_{w}}$ $=0.66 \cdot 10^{6}$ (calculated on the free-stream flow parameters and bluntness radius of sphere $R_{w}=37.5 \mathrm{~mm}$ ), flow stagnation temperature $T_{0}$ $=573 \mathrm{~K}$, and pressure behind the normal shock $p_{s}=0.5$ bar. The mass concentration of solid particles in the flow did not exceed $3 \%$. The cooling gas was injected in the dusted flow through the tangential axissymmetric slot between a flat "cap" and the sphere surface. The blown gas rate was $G^{*}=G_{j} /\left(\pi \rho_{\infty} u_{\infty} R_{w}^{2}\right)=0 \ldots 0.12$. It is shown that to decrease the maximum of heat flux toward the sphere surface in two times, the mass flow rate of coolant $G^{*}=0.03$ is needed.

\section{NONSTANDARD NOMENCLATURE}

\author{
Variables \\ $d_{m} \quad$ mean weight diameter of particles \\ e particle inertia \\ $m_{i} \quad$ weight fraction \\ $n \quad$ weight particles concentration \\ $R_{w} \quad$ blunted body radius \\ $\Psi \quad$ correction coefficient
}

This is an Open Access article distributed under the terms of the Creative Commons Attribution-Noncommercial License 3.0, which permits unrestricted use, distribution, and reproduction in any noncommercial medium, provided the original work is properly cited. 


\section{Indices}

c critical point

is isentropic braking

$m$ middle (averaged)

$p$ particle

$s$ behind the normal shock

$w$ body surface

$0 \quad$ without particles

\section{INTRODUCTION}

Dusty flow over bodies is essentially much more complicated comparing with a pure gas flow. On the body surface in a dense flow, the gas stagnation occurs and the conditions of gas sticking to the body surface in flow are realized as a rule. On the other side, due to inertia of dust particles, their trajectories can differ essentially from gas stream lines. When the particles are large and dense, they practically do not lose the speed near the body surface. Such particles, as a rule, when interacting with the body surface, transfer part of their energy to the body and reflect from it. The erosion [1] or the particles pickup with the creating of the strong coating [2] can take place at particles high speed nearby the surface body. The deceleration of fine particles of low-density substance in a compressed gas layer can be practically total. The particles are not deposited on the body surface in this case.

Such interactions of a high-speed dusty flow with a blunt body arise at a high-speed motion of bodies in dusty atmospheres (e.g., Mars atmosphere), in elements of solid-propellant engines, at chambers of power stations and chemicals industries, at the spray application, during material cutting by a supersonic dusty fluid, and in many other cases.

When a body is in gas flow with condensed particles, a variety of physical effects can occur [3-10]: significant electrization of the body surface and particles (to several kilovolt and more) [3, 7]; increasing of particle concentration near the front body surface by orders of magnitude; powerful optical radiation near the body surface; sticking of particles to the body surface, penetration of particles into the external body layer or intense erosion of the body surface [5]; increase in heat transfer between a dusty gas flow and a body in flow by many times $[5,6,9]$.

A lot of works are devoted to the methods of thermal protection of front body surface streamlined by high-temperature clean gas. A significant part of them concerns to the research of thermal protection by gas injection in the boundary layer. It was shown that continuously distributed injection (for example, through a porous wall) is the most effective method. However, this way is not reliable because of unpredictability and instability of hydraulic characteristics of porous materials. Injection through perforated orifices is much less effective 
in comparison with injection through the porous material. In some cases, the injecting through perforated orifices can result even in increase of heat flux.

A simple and effective method for heat protection of the front surface of the blunted body is injection through a tangential slot [4]. In the present work, the tangential injection is investigated as a mean of thermal protection of the front surface of body against acting of high-temperature dusty gas.

\section{MODEL}

The model represents itself a spherically blunted cylinder (Fig. 1). The cooling gas is supplied in the model through a central tube. The tangential axissymmetric slot is created between a flat "cap" and the sphere surface. The slot height $h_{k}$ can be changed by rotation of the stem "cap" with very small thread in the central tube. Because of sphere curvature, the slot height in the exit section $h$ is bigger than in the minimal entrance section $h_{k}$. Thus, the ratio of the areas in critical and exit sections of the slot is $F_{k} / F_{e}=\left(d_{k} \times h_{k}\right) /\left(d_{e} \times h_{e}\right)$. When the minimal slot height is $h_{k}=0.2 \mathrm{~mm}$, the calculated Much number at the slot exit is $\mathrm{M}=2.56$, and at the slot height $h_{k}=0.8 \mathrm{~mm}, \mathrm{M}=2.04$. In all cases, the injected flow was supersonic that guaranteed uniform distribution of coolant in the azimuth direction.
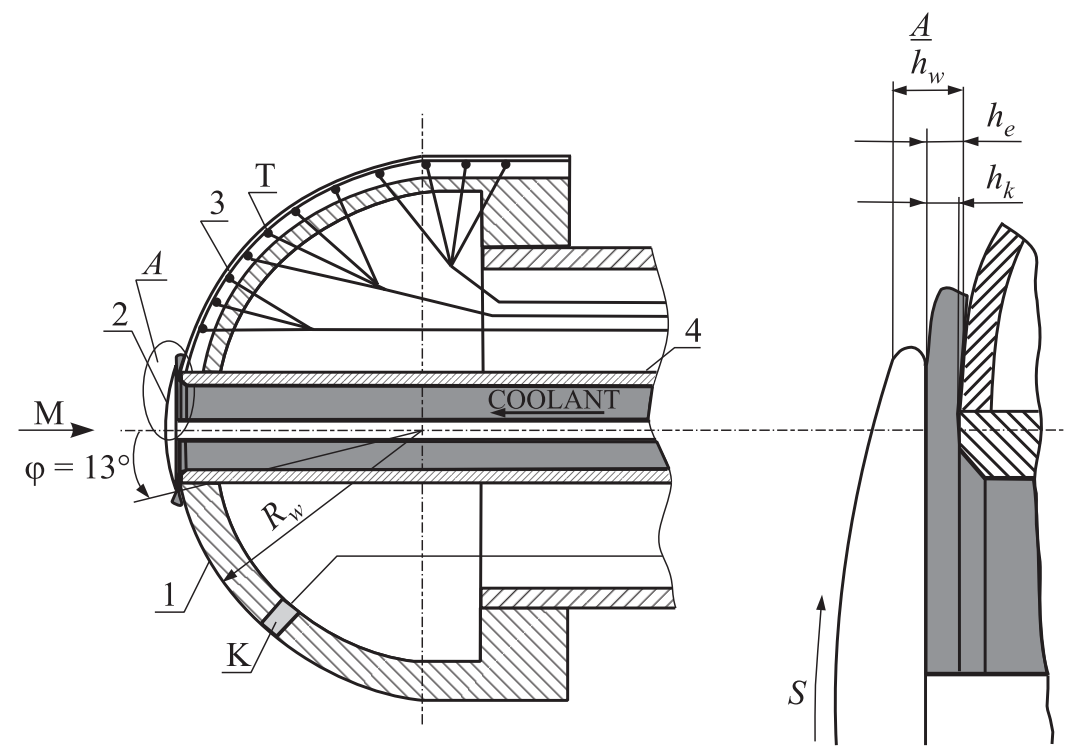

Figure 1 The model with the tangential slot on the spherical nose: $1-$ nose; $2-$ cup of slot; 3 - thin wall; 4 - coolant channel; $\mathrm{T}$ - thermocouples; $\mathrm{K}$ - calorimeters; and $h_{k}-$ slot 
In experiments, gas supplying in the model was carried out by means of the fast-working system.

The cross section of each element of the system exceeds essentially the minimal cross section of the slot. Therefore, gas velocity in the system was much lower than the sound velocity in the slot where height was maximum $h_{k}=0.8 \mathrm{~mm}$.

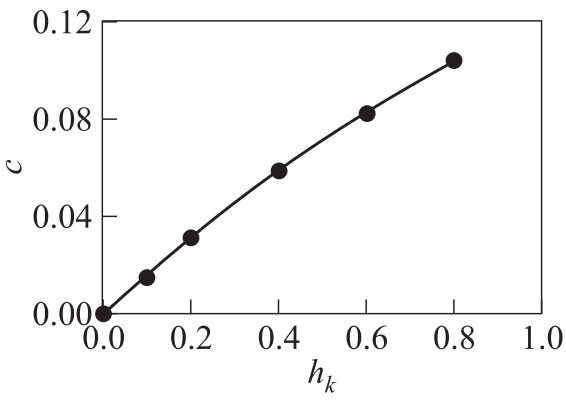

Figure 2 Hydraulic resistance of the slot $c$ depending on slot height $h_{k}$

Tests of the system together with the model have shown that hydraulic resistance of the supply system is less than $1 \%$ of the model hydraulic resistance.

At the tests in the wind tunnel, the supply of cooling gas has begun before the diaphragm rupture with an advancing $\tau=0.1 \ldots 0.5 \mathrm{~ms}$ and was stopped after the end of heat flux measurements. Pressure of blown gas was $P_{j}=2 \ldots 32$ bar (at stagnation pressure $P_{s} \leq 0.5$ bar). The blown gas rate was $G_{j}=0 \ldots 4 \mathrm{~g} / \mathrm{s}$ $\left(G_{j}=0\right.$ at $\left.h=0\right)$ that corresponds to the dimensionless flow rate $G^{*}$ $=G_{j} /\left(\pi \rho_{\infty} u_{\infty} R_{w}^{2}\right)=0 \ldots 0.12$ where $\rho_{\infty}$ and $u_{\infty}$ are the density and velocity of wind tunnel free flow, respectively. Before the experiments in the wind tunnel, careful measurements of blown gas rate $G_{j}$ in dependence on pressure $P_{b}$ have been carried out.

The injected gas rate was defined by the formula:

$$
G=\frac{c\left(h_{k}\right) P_{b}}{T_{b}^{0.5}}
$$

where $P_{b}$ is the pressure in the balloon; $T_{b}$ is the cooler temperature ahead the model; $c\left(h_{k}\right)$ is the experimentally established dependence of hydraulic resistance of the slot on its height $h_{k}$ (Fig. 2). Duration of the experiments at hydraulic tests essentially exceeded duration of tests in the wind tunnel. Due to this, the error in injected gas rate measurement did not exceed $3 \%$.

There were used the thin wall technique and calorimeters for measuring of heat flux on a body surface. A stainless steel foil band $21 \mathrm{~mm}$ in width and $0.2 \mathrm{~mm}$ in thickness was welded flush to the model surface at using thin wall technique. On the inside, 0.1-diameter wires from kopel alloy were welded to the foil. Near the welding point, the wires were expanded up to the thickness of $0.030-0.04 \mathrm{~mm}$. The dimensions of the thermocouple contact zone were 0.2 $\times 0.2 \mathrm{~mm}$.

In the thin wall technique, the heat flux is usually obtained from the relationship for one-dimensional heat conduction: $q=k d T / d \tau$, where $k \approx \rho c \delta$ is 
the calibration coefficient; $\rho$ and $c$ are the density and heat capacity of the wall material; $\delta$ is its thickness; $T$ is the temperature; and $\tau$ is the time.

The coefficient $k$ is determined using the calibration device. The numerical calculations carried out with consideration of two-dimensional heat conduction and the experiments with the walls of different thicknesses $(\delta=0.1$ and $0.2 \mathrm{~mm})$ reveal that lateral heat conduction along the wall exerts a weak influence on the measurement results in the conditions under study.

\section{SUPPLY SYSTEM OF THE INJECTED GAS INTO THE MODEL}

Basic elements of this system are (Fig. 3): a balloon in 19 capacities about $40 \mathrm{l}$; gate 17 for filling a balloon; valve of dump of pressure of gas in an atmosphere 18;

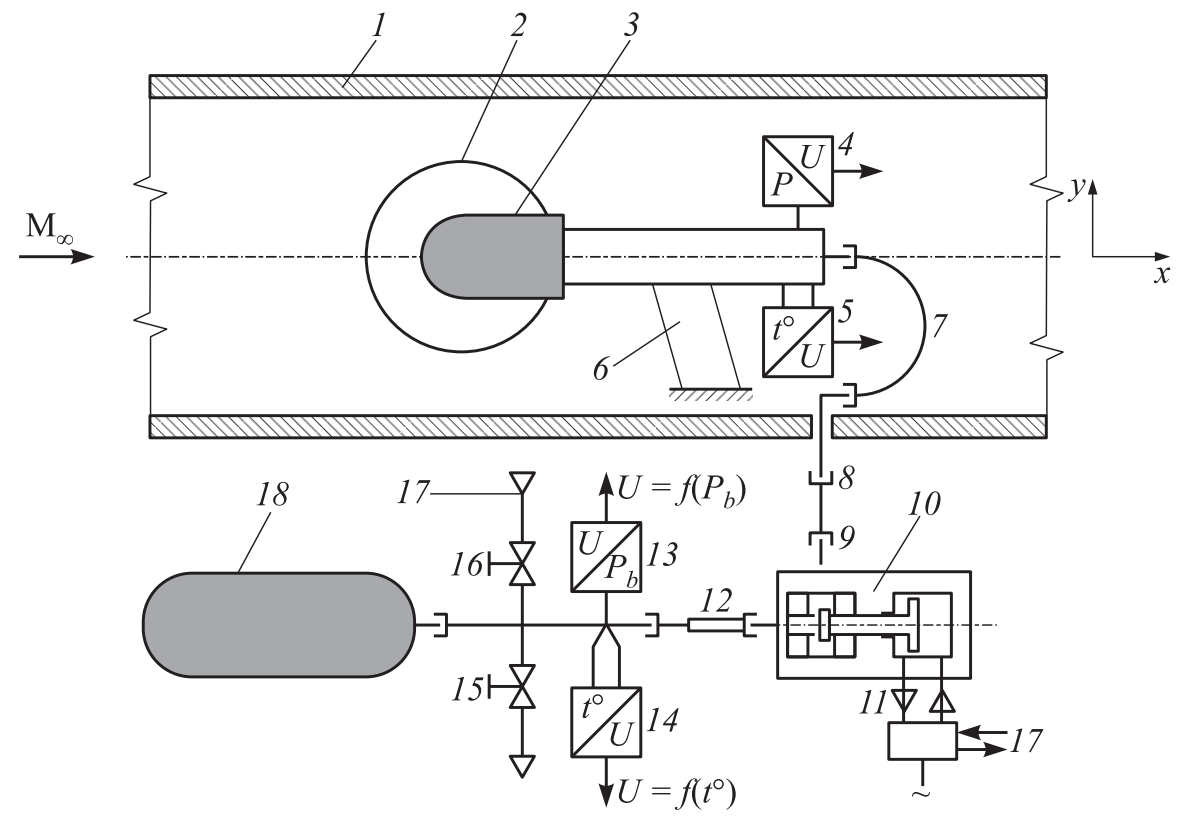

Figure 3 The supply system of injected gas into the model: 1 - walls of test section; 2 - optical window in UT-1 test section; 3 - blunt body; 4 - pressure gauge at model inlet; 5 - temperature gauge at model inlet; 6 - pylon of model; 7,8 , and 12 - gas supply pipes; 9 - electrical quick-response pressure transducer; 10 main pneumatic-electrical valve; 11 - control electrical valve; 13 - vessel pressure gauge; 14 - vessel exit temperature gauge; 15 - vessel pressure release valve; 16 vessel filling valve; 17 - high-pressure tube; and 18 - vessel 
and pneumoelectric high-speed valve 10. The maximal gas pressure in the supply system is 150 bar. Time necessary for pressure establishment in the model after valve opening makes approximately $0.2 \mathrm{~ms}$. Pressure $P_{j}$ and temperature $T_{j}$ in the supply gas system to model are registered both on an output from a balloon (gauges 15 and 16, respectively) and on an input in the model (gauges 8 and 9 ).

The gauge of pressure 8 has small inertia $(t \sim 2 \mathrm{~ms})$. The junction of thermocouple wires of the gauge was settled down on an axis of the bringing channel 10. Inertia of the temperature gauge 9 was approximately the same, as well as of the pressure gauge.

\section{CREATING OF DUSTED FLOW IN THE WIND TUNNEL}

The high pressure channel of the wind tunnel with the inner diameter of $70 \mathrm{~mm}$ and length 6 or $12 \mathrm{~m}$ was equipped by an external ohmic heater which provides gas heating up to $T_{0}=800 \mathrm{~K}$ (Fig. 4). The test section diameter was $2 R_{n}$ $=0.5 \mathrm{~m}$. Duration of the steady-state flow was $\tau=23$ or $46 \mathrm{~ms}$.

For introducing the particles into the flow, the "fluidized bed" method was used. As a rule, the mixer (Fig. 5) was installed in one inlet used to introduce the dusty gas into the duct wind tunnel entry 1 located near diaphragms 2 of the UT-1 tunnel. The inner channel of the mixer is shaped as a vertical cone with the half apex angle of $15^{\circ}$ and $71 \mathrm{~mm}$ high. The cone outlet diameter is $41.2 \mathrm{~mm}$.

At the outlet section of the cone, a perforated membrane was mounted with hole diameter of $2 \mathrm{~mm}$ and the step between holes of $5 \mathrm{~mm}$. The mixer volume was $36 \mathrm{~cm}^{3}$. At the beginning of an experiment, volume of the dust was from 1 to $10 \mathrm{~cm}^{3}$.

The estimation have shown that even at maximum duration of the steadystate flow, the air flowing through the nozzle occupies only the front part of the

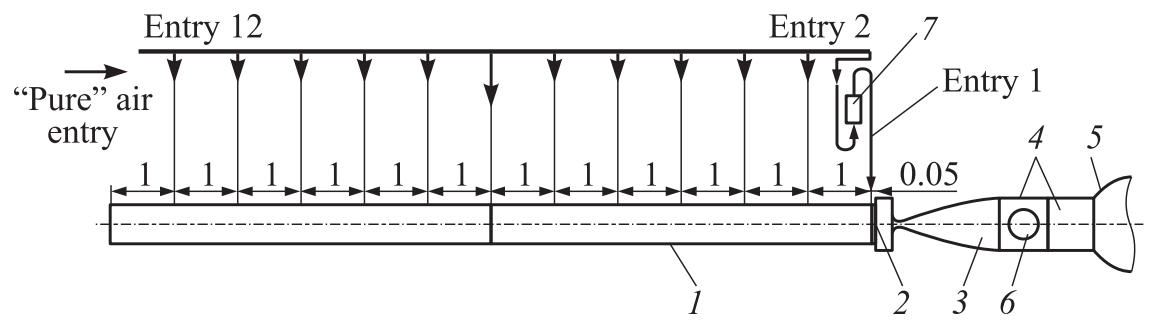

Figure 4 Wind tunnel UT-1: 1 - duct; 2 - diaphragm; 3 - nozzle; 4 - test section; 5 - exhaust chamber; 6 - optical window; and $7-$ mixing device. Dimensions are in meters 


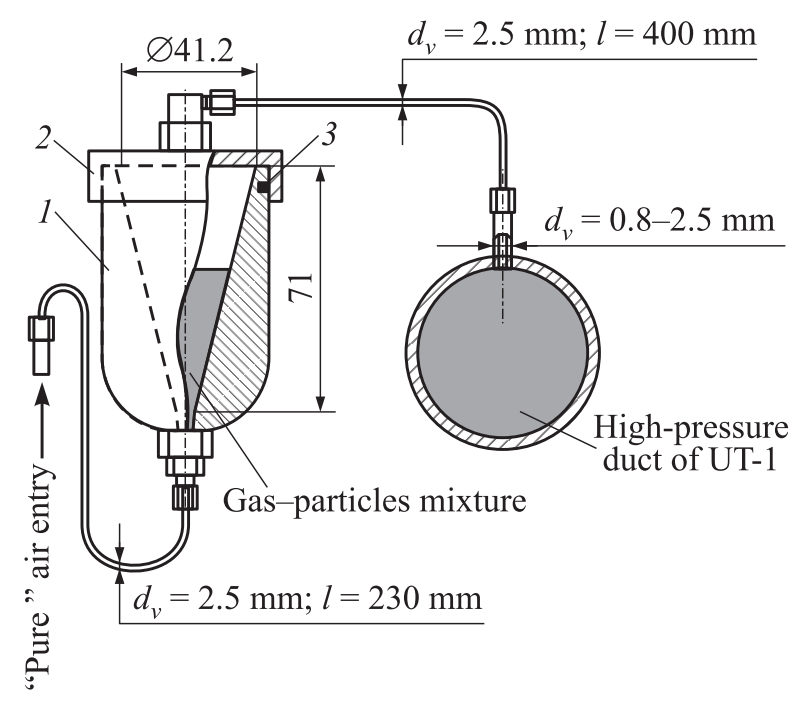

Figure 5 Mixing device. Dimensions are in millimeters

high-pressure channel (about $3 \mathrm{~m}$ in length) situated near the diaphragm and the nozzle. Therefore, it is expedient to fill by the dust only the front part of the channel. At the end of each test (at $\tau \geq 46 \mathrm{~ms}$ ), halfway cleaning of the front channel from the particles by "clean" air expiring from rear part of the channel was performed. If the tests conditions do not change, such test technology provides good repeatability of particles concentration and heat exchange beginning from the third test.

To examine the particle parameters in the nozzle flow, a quasi-onedimensional dusty-gas flow in the internal duct of the wind tunnel UT-1 was simulated numerically. Calculations have shown that for the particles used, their thermal protection and speed of sliding relatively the carrying phase did not exceed $2 \%$.

For investigation of free dusted gas flow and particles flow near the body surface, it was used both a shadow method and a method based on registration of scattered light. As an example, Fig. 6 presents a scheme in which the light sheet ("laser knife") was used.

The radiation intensity distribution in cross section of the free flow (ahead the bow shock wave) is presented in Fig. 7. At small volumetric concentration of particles, intensity of the scattered light is proportional to the particle concentration, and the ratio of intensities of two wavelengths $I_{r} / I_{b}$ characterizes the mean particle size. This data show that the concentration distribution is approximately uniform in the jet core which is $150 \mathrm{~mm}$ in diameter. The intensity 


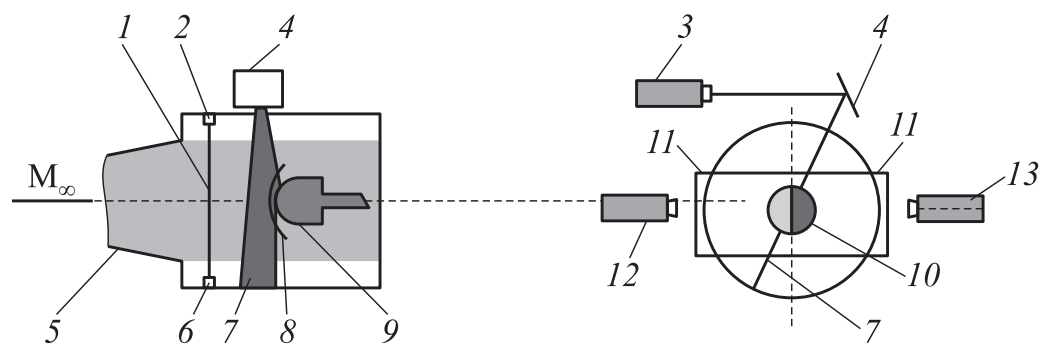

Figure 6 Optic scheme: $1-$ continuous wave laser; $2-$ continuous laser; $3-\mathrm{Nd}$ : YAG laser; $\lambda=0.53,1.06 \mu \mathrm{m} ; 4$ - mirror; 5 - nozzle; 6 - beam dump; 7 - light sheet; 8 - bow shock; 9 - model; 10 - shadow surface model; 11 - optical window; 12 - videocamera; and 13 - photocamera

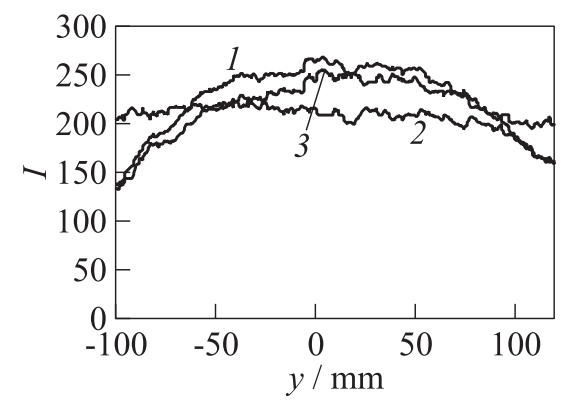

Figure 7 Distribution of light intensity in infrared $\left(I_{r}\right)(1)$ and green $\left(I_{b}\right)(2)$ intervals and ratio $I_{r} /\left(I_{b} \times 200\right)(3)$ in cross section of free flow

ratio of the diffused light $I_{r} / I_{b}$ in the jet core is approximately uniform too. It testifies that the average size of particles in the cross section of the jet core is also approximately constant.

The presented data are obtained at short exposition which is approximately $10^{5}$ times smaller than the characteristic sensitivity time $\tau_{\text {cal }}$ of the calorimetric gauges used.

Estimations show that concentration alterations during the period of $\tau \approx \tau_{\text {cal }}$ do not exceed some percents.

The particles concentration was registered during a run by the red-color photodiode laser (wave length of $0.65 \mu \mathrm{m}$ ) with the power of $2 \mathrm{~mW}$. The laser was located near the upper surface of the test section. By means of a mirror, the light beam crossed the gas flow from top to bottom. Near the bottom surface of the test section, a "light trap" was mounted which reduced intensity of the light reflected from the surface of the test section. To transform the photoelectron multiplier (PEM) signal into the particle weight flow, it was assumed that: 


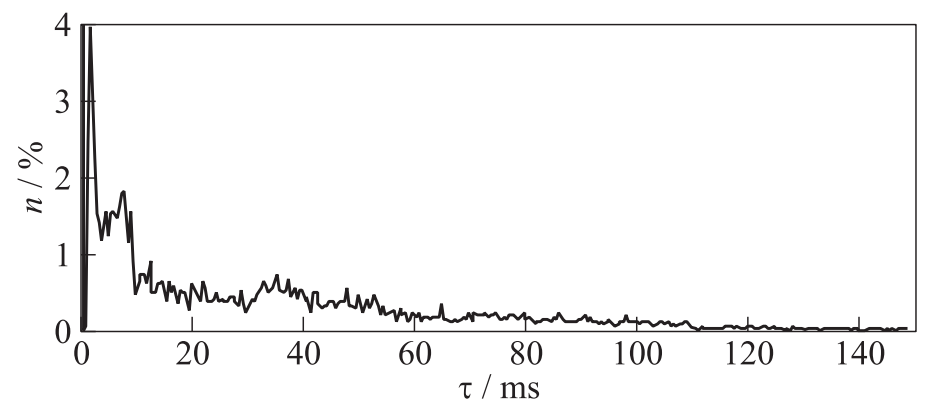

Figure 8 Weight concentration of particles in the jet core $n$ during run

- particles weight flow is directly proportional to the signal recorded;

- particles concentration is constant across the jet section; and

- total powder amount in the mixer before the test is entrained by the gas flow to the end of registering the test parameters.

With account of these assumptions, one can write:

$$
G_{p}=k \int_{0}^{\tau} J d \tau
$$

where $G_{p}$ is the powder weight in the mixer before the test; $J$ is the current value of the signal; and $\tau$ is the current time. This formula was sued for determining the value of the coefficient $k$ for a given test. The current particles concentration was determined from the formula:

$$
n_{\tau}=\frac{k J}{\pi \rho_{\infty} u_{\infty} R_{n}^{2}} .
$$

The example of registration with the help of the given system is shown in Fig. 8. It is shown that the particles concentration during $10 \leq \tau \leq 46 \mathrm{~ms}$ has approximately the constant value.

\section{PARTICLES}

In the intermediate range of size and density of particles, their deceleration in a disturbed gas flow occurs only partially. The extent of deceleration of particles and the direction of their collision on the surface are also influenced by the shape of particles, the size and shape of a body immersed in flow, density and viscosity 
of carrying gas flow, and some other characteristics of particles and carrying gas flow.

The main dimensionless parameter characterizing the translational motion of particles in gradient flow over a blunted body is the particle inertia:

$$
e=\frac{d_{p}^{2} u_{\infty} \rho_{p} \Psi}{27 \mu_{s} R_{w}} .
$$

Here, $d_{p}$ and $\rho_{p}$ are the particle diameter and density; $u$ is the speed; $\mu$ is the gas viscosity; $R_{w}$ is the blunted body radius; indices $\infty$ and $s$ refer to undisturbed flow and to flow behind the normal shock wave; $\Psi=1+\operatorname{Kn}(2.492$ $+0.84 \exp (-1.74 / \mathrm{Kn}))$ is the correction coefficient taking account of the Knudsen number effect on the Stokes particle drag: $\mathrm{Kn}=L_{s} / D_{p}$, where $L_{s}$ is the free path length of gas molecules behind the shock wave:

$$
L_{s}=\frac{1.255 \mu_{s}}{\left(\rho_{s} R_{i} T_{s}\right)^{0.5}} ;
$$

$R_{i}$ is the gas constant; and $\mu_{s}$ and $T_{s}$ are the gas density and temperature behind the normal shock wave.

Note that particles in flow (as in atmosphere) can be of very small size tens of micron and less. Hence, the correction coefficient $\Psi$ for such particles can significantly differ from 1.

In the authors' experiments, the particles $\mathrm{Fe}_{2} \mathrm{O}_{3}$ having $d_{m}=0.37 \mu \mathrm{m}$ and moderate inertia $e=0.25$ were used (here, $d_{m}=\sum d_{i} p_{i}$ ).

The particle inertia is calculated for experimental conditions in the wind tunnel: the free-stream Mach number $\mathrm{M}_{\infty}=6$, the Reynolds number $\operatorname{Re}_{\infty, R_{w}}$ $=6.6 \cdot 10^{5}$ (based on the free-stream flow parameters and bluntness radius of

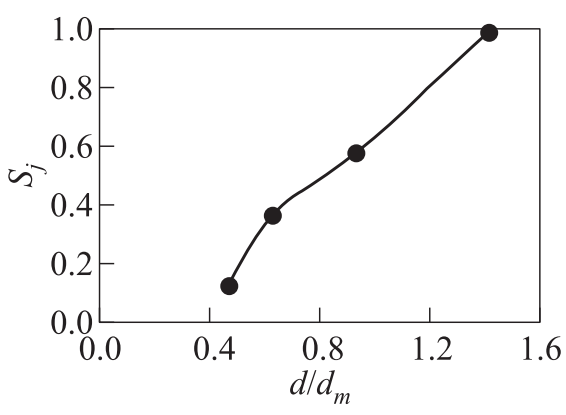

Figure 9 The particle weight distribution sphere $\left.R_{w}=37.5 \mathrm{~mm}\right)$, the flow stagnation temperature $T_{0}=573 \mathrm{~K}$, and the total isentropic pressure $p_{i s}$ $=20$ bar (pressure behind the normal shock $p_{s}=0.51$ bar). The particle weight distribution $S_{j}$ $=\sum_{j}\left(d_{i} p_{i} / d_{m}\right)$ as a function of the nondimensional particle diameter $d / d_{m}$ is shown in Fig. 9 .

When the particle inertia is small (equal or less than $\leq 0.12$ ), the particles are totally decelerated in a compressed gas layer ahead of the body and do not deposit on its surface. However, they transfer the part of their kinetic energy to the gas layer at the boundary layer bottom and increase heat transfer. 


\section{EXPERIMENTAL RESULTS}

The calculations and experiments have revealed that even when the inertia of particles is so low that they are not deposited on the body surface and with their small concentration in an undisturbed gas flow (about $1 \%$ (wt.)), the effect of particles on heat exchange between the dusty gas flow and the body surface can be significant.

In example in Fig. 10, the ratio of heat flux in the given point of the surface to the calculated heat flux at critical point of body for smooth surface at laminar boundary layer (curve 1 ) is shown. Experimental results at pure gas at near critical point exceed the

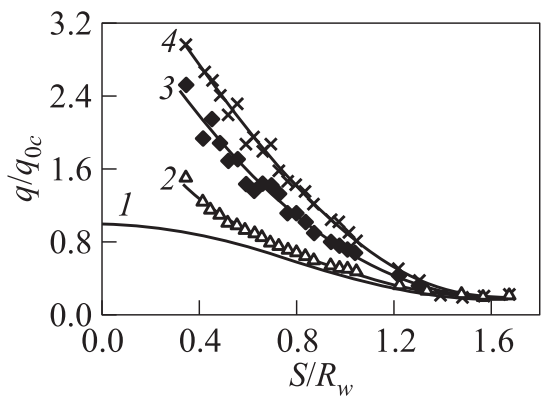

Figure 10 The ratio of heat flux in the given point of the surface to the calculated heat flux at critical point of body: 1 - for smooth surface at laminar boundary layer; $2-n_{m}=0 \% ; 3-1.4 \%$; and $4-n_{m}$ $=2.6 \%$ calculated value. This effect is due to the fact that the dripping boundary layer from "cap" (see Fig. 1) is appended to the sphere surface. The presence of particles $\mathrm{Fe}_{2} \mathrm{O}_{3}$ at small weight concentration sufficiently increases the heat flux to the body surface.

Figure 11 illustrates the ratio of the heat flux obtained in the experiments with particles $q$ to the heat flux at the same surface point but without particles (in pure flow) $q_{0}$ on the model surface. It is seen that on the whole, the front surface of the sphere bluntness is as far as the central angle of about $80^{\circ}\left(S / R_{w}=1.4\right)$. At the central angle less $40^{\circ}\left(S / R_{w}=0.7\right)$, the increase in the heat flux is constant: $q / q_{0}=1.7$ at concentration $n_{m}=1.4 \%$ (curve 2 ) and $q / q_{0}=2.2$ at concentration $n_{m}=2.6 \%$ (curve 4 ).

Such noticeable effect of particles with low inertia (small particle size with respect to the body size) on heat exchange is caused by the fact that the particles lose a significant part of their kinetic energy in the boundary layer of gas and increase the flow enthalpy near the body surface in flow. Besides, the particles transfer the attached high-temperature gas flow mass to the boundary layer part adjacent to the surface and also cause its turbulization.

The heat flux distribution on the front spherical surface of the model is shown in Fig. 12 for various conditions: in the pure flow and at presence of solid particles $\mathrm{Fe}_{2} \mathrm{O}_{3}$. The flow rate of cooling air varied from $G^{*}=0$ (in this case, the slot has been hermetically sealed) up to the maximal $G^{*}$ value. One can see that in the flow of "pure" gas, the heat flux is much less than in the flow of the dusty gas.

Even at small relative flow rate of the blown gas, the heat flux $q$ near the slot decreases essentially. Moreover, the heat flux immediately at the slot exit is 


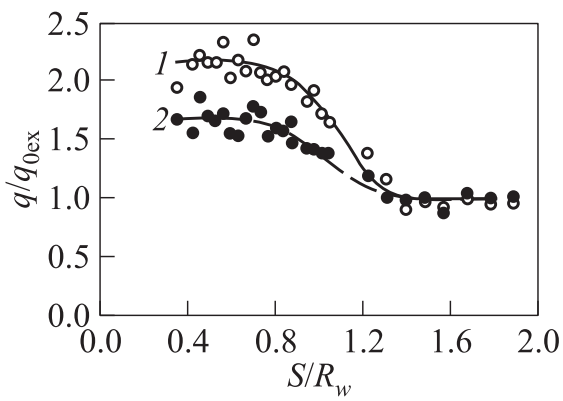

Figure 11 The ratio of heat flux in the given point of the surface at the dusted flow to the heat flux in this point at the clean gas flow. Injection is absent: $1-$ $n_{m}=2.6 \%$; and $2-n_{m}=1.4 \%$

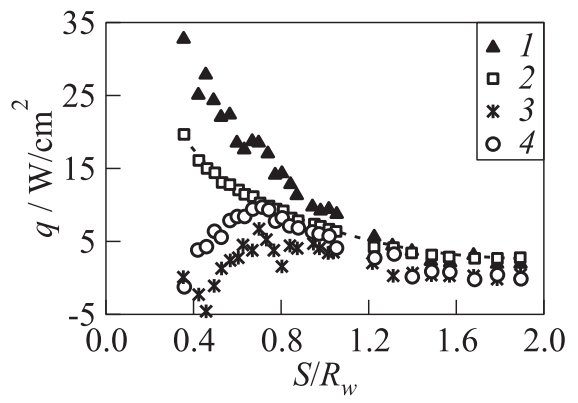

Figure 12 Distribution of heat flux on the cylinder with the spherical nose: $1-$ $n_{m}=1.4 \%, G^{*}=0 ; 2-n_{m}=0 \%$, $G^{*}=0 ; 3-n_{m}=1.4 \% ; G^{*}=0.15 ;$ and $4-n_{m}=1.4 \%, G^{*}=0.075$

directed from the wall to the gas, i. e., has negative value. This is because the static temperature of the blown gas in this zone is lower than the model surface temperature. Increase of mass flow rate $G^{*}$ results in additional decrease of the heat flux. At the maximal mass flow rate, the level of the maximal heat flux appreciably goes down, too.

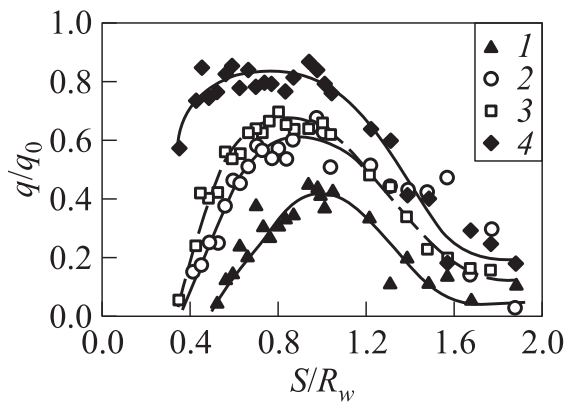

Figure 13 The ratio of the heat flux at coolant injection rate $G_{\text {inj }}$ to heat flux without injection; $\operatorname{Re}_{\infty, R_{w}}$ $=0.38 \cdot 10^{6} ; q_{0}$ - heat flux at $G^{*}=0$; the weight concentration $n_{m}=1.4 \%$; $G^{*}=G_{\text {inj }} /\left(\rho_{\infty} u_{\infty} R_{w}^{2}\right)-$ coolant rate (air): $1-G^{*}=0.15 ; 2-0.075 ; 3-$ 0.035 ; and $4-G^{*}=0.019$

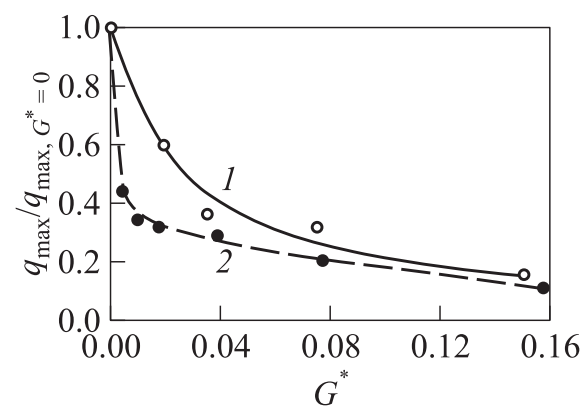

Figure 14 Influence of mass rate injection $G^{*}$ on heat flux maximum $q_{\max } / q_{\max , G^{*}=0}: 1-n_{m}=1.6 \%$; and $2-n_{m}=0 \%$ 
The heat flux at air injection related to the heat flux at the same point at absence of injection is presented in Fig. 13 in dependence on distance from the stagnation point. In Fig. 14, the results of experiments with particles $\mathrm{Fe}_{2} \mathrm{O}_{3}$ are presented: the maximal heat flux values $q_{\max }$ received at injection of cooling gas referred to the similar data $q_{\max , G=0}$ received without injection are depicted in dependence on the relative mass flow rate of blown gas $G^{*}$.

On the basis of the received experimental results, it is possible to conclude that the heat flux to the front surface of a blunt body streamlined by a supersonic flow of dusty high-temperature gas can be essentially reduced by injection of cooling gas through the tangential slot located near the body stagnation point. As an example, in order to decrease the maximum of heat flux towards the sphere surface in two times, the mass flow rate of coolant $G^{*}=0.03$ is needed merily.

\section{CONCLUDING REMARKS}

On the basis of the received experimental results, it is possible to conclude that the heat flux to the front surface of a blunt body streamlined by a supersonic flow of dusty high-temperature gas can be essentially reduced by injection of cooling gas through the tangential slot located near the body stagnation point.

\section{ACKNOWLEDGMENTS}

This work was carried out at the support of the Russian Foundation for Basic Research (grants 07-01-00678-a and 09-08-00542-a).

\section{REFERENCES}

1. Mikhatulin, D., Y. Polezhaev, and L. Reviznikov. 2005. Thermo-erosion destruction of materials in supersonic heterogeneous flows. European Conference for AeroSpace Sciences Proceedings. July 4-7, 2005. Moscow. CD. No. 4.04.06. P. 1-9.

2. Nikitin P. 1994. Cold gas dynamic method for synthesis of the composite materials and multifunctional coating with the predicted thermophysical characteristics. Conference (International) on Fluid and Thermal Energy Conversion '94 Proceedings. Denpasar, Bali, Indonesia. December 1994.

3. Kolecki, J.C., B. Hillard, and M. Sielbert. 1996. Overview of Mars system environment interactions. AIAA Paper No. 96-2333.

4. Borovoy, V. Ya., Ed. B. Vasilevsky, I. V. Struminskaya, and L. V. Yakovleva. 1998. Gas flow and heat protection by strong injection in the shock wave interference region near the blunt body front surface. 3rd European Symposium on Aerothermodynamics for Space Vehicles, ESA SP-426 Proceedings. 501-7. 
5. Vasilevskii, E. B., and A. N. Osiptsov. 1999. Experimental and numerical study of heat transfer on a blunt body in dusty hypersonic flow. AIAA Paper No. 99-3563. $1-11$.

6. Vasilevskii, E. B., A. N. Osipov, A. V. Chirikhin, and L. V. Yakovleva. 2001. Heat exchange on the front surface of a blunt body in a high-speed flow containing low-inertia particles. J. Eng. Phys. Thermophys. 74(6):34-42.

7. Vasilevskii, E., V. Gorelov, R. Kazansky, and L. Yakovleva. 2002. Body electrization in a supersonic two-phase flow. 2nd Conference (International) "Materials and Coating for Extreme Performances: Investigations, Applications, Ecologically Safe Technologies for Their Production and Utilization" Proceedings. 16-20 September, 2002. Katseveli-town, Crimea, Ukraine. 23-24.

8. Vasilevsky, E. B., L. A. Dombrovsky, D.S. Mikhatulin, et al. 2002. Heat transfer in a heterogeneous supersonic flow. 12th Heat Transfer Conference (International) Proceedings. August 18-23, 2002. Grenoble, France. 3:177-81.

9. Vasilevskii, E. B., V. P. Kulesh, Yu. V. Zhilin, and L. V. Yakovleva. 2004. Diagnostics of supersonic dusted flow near a blunt body. 12th Conference (International) on the Methods of Aerophysical Research Proceedings. 28 June-3 July, 2004. Novosibirsk, Russia. 299-304.

10. Vasilevskii, E. B., T. V. Ershova, D. S. Mikhatulin, and L. V. Yakovleva. The physical effects arising at interaction of a high-speed dusty flow with a blunted body. 2007. 2nd European Conference for AeroSpace Sciences (EUCASS) Proceedings. July 1-6, 2007. Brussels. 